\title{
NOTES
}

\section{The Commodore 64 and an interface system for controlling operant chambers}

\author{
JERRY W. O'DELL and DONALD E. JACKSON \\ Eastern Michigan University, Ypsilanti, Michigan
}

This article describes the interfacing of a Commodore 64 computer with an operant chamber. Advantages of the Commodore 64 over the VIC-20 as a controller are listed, and a sample program using dual response levers is given.

Recently, Jackson and O'Dell (1985) described the use of a VIC- 20 computer as a controller for operant chambers. The Commodore 64 , selling for under $\$ 140$, has advantages over the VIC-20 that justify its consideration as the controller of choice. The Commodore 64 has a greater number of characters per line on the screen, much more memory, better graphics, far superior sound, two counters, two time-of-day clocks, and other features. In this paper we describe a way to use the Commodore 64 as a controller, and we also provide information on implementing the second counter, an important consideration for those with dual-lever chambers.

As with the VIC-20, a television monitor and a data cassette recorder (currently available at K-Mart for \$20) are needed. Used and new 12-in. television monitors are inexpensive.

\section{Overview of the Interface and Control}

The system uses the $28-\mathrm{V}$ power supply, power rods, and lever control and connection panels from the original Lehigh Valley 30,000-series equipment. Such original equipment, or similar equipment, is necessary so that switch closures on the interface result in delivery of $28 \mathrm{~V}$ to cue lights, houselight, food magazine, lever extend/retract motor, and so forth.

A card-edge connector plugs into the user port on the back of the Commodore 64 . Wires from this connector (TRW 50-24A-30) run to a protoboard (276-169 Archer Universal Breadboard from Radio Shack) that contains the low-level TTL circuitry. Wires from the protoboard run to a locally constructed $\mathrm{I} / \mathrm{O}$ panel that is mounted on the power rods of the Lehigh Valley system. This panel contains the optoisolators, light-emitting diodes (LEDs), power transistors, diodes, and output studs needed to drive large electrical loads. In addition, a 5-V regulated power supply is required to run the low-level circuitry.

The Protoboard. The Universal Breadboard is relatively inexpensive (\$19.95) and allows easy alteration if

\footnotetext{
The authors' mailing address is: Department of Psychology, Eastern Michigan University, Ypsilanti, MI 48197.
}

needed (e.g., the addition of OR gates). The breadboard holds the integrated circuit (the 7400 in Figure 1) used to transfer inputs from the operant chamber (switch closures) to the computer. It also contains the 7406 integrated circuits needed to transfer outputs from the Commodore 64 to the power circuitry, and then only to those devices (cue lights, houselight, feeder, and lever extend/retract motor) which require $28 \mathrm{~V}$. To protect the computer, power to this board should come from an independent 5-V power supply. Such a power supply can be obtained for $\$ 14.95$ from Jameco Electronics, Belmont, California, or can be built from easily obtained parts for about $\$ 10$.

Input Circuitry. The input circuitry from the operant lever consists of a 7400 integrated circuit mounted on the Universal Breadboard. This circuit senses and debounces switch closures in the operant chamber via the studs on the lever-control panel. The 7400 output enters the computer via the I/O user port on the back of the computer. As shown in Figure 1, output from the left lever should go to pin-location 4 and output from the right lever should go to pin-location 6 on the card-edge connector. This configuration allows independent counts from the two response levers. Table 1 lists a computer program to set and read the counters.

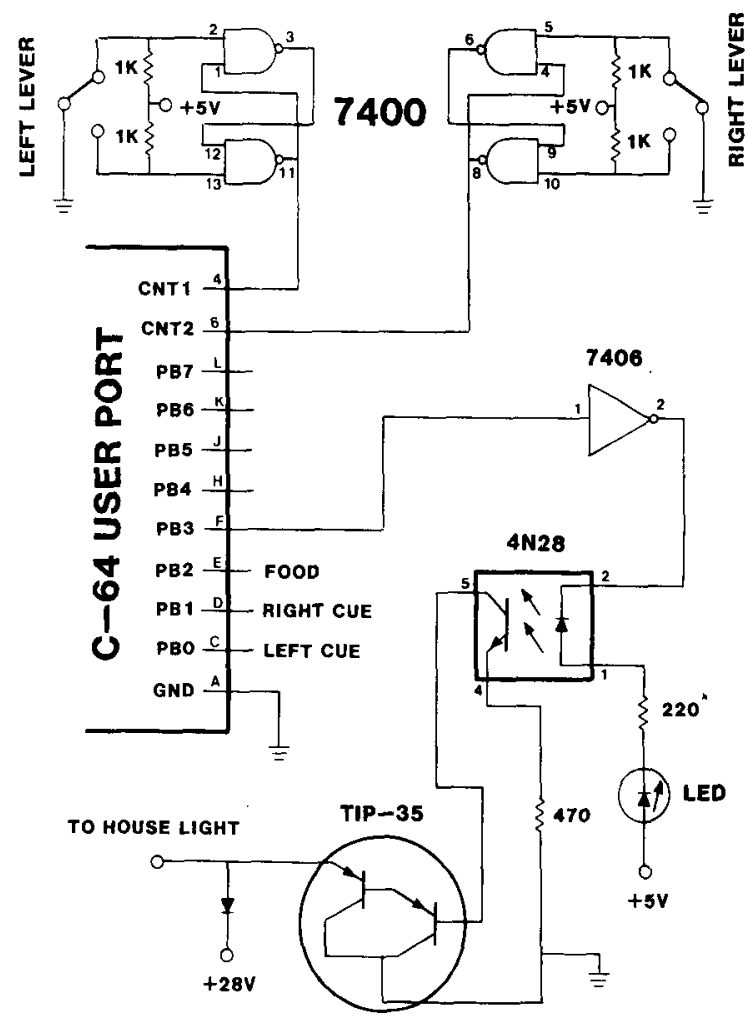

Figure 1. Circuit diagram. 
Output Circuitry. Since counts are input from pinlocations 4 and 6 of the user port, the remaining eight lines can be used for output. Although only one output circuit is shown in detail in Figure 1, all other outputs have the same circuitry.

The TTL output signals from the computer enter one of the two 7406 integrated circuits on the Universal Breadboard. Output from this inverter is routed through an optoisolator, a $4 \mathrm{~N} 28$, which then controls the power transistor. The purpose of the optoisolator is to electrically separate the delicate computer components from the power-handling portions of the circuit. A TIP-135 PNP Darlington transistor is used to provide sufficient power to drive external loads. Use of the TIP-135 allows any of the eight lines to handle the large current requirements of the feeder solenoid. The design of the $\mathrm{I} / \mathrm{O}$ panel ensures adequate heat dissipation from the transistors. Diodes protect the transistors from the inductive kick of the feeder or other solenoid coils. This circuit is shown on the bottom part of Figure 1.
These output circuits are mounted on a panel that clips directly onto the power rods of the old Lehigh Valley system. The bottom portion of the panel consists of an L-shaped piece of heavy aluminum, to which the collectors of the TIP-135 power transistors are bolted. The aluminum serves as a heat sink. The upper portion of the panel is a $6 \times 8$ in. piece of "perf-board" (Archer 1761396), on which the optoisolators, LEDs, and resistors for the circuit are mounted.

\section{A Simple Experiment and Program}

The following experiment demonstrates how to use both response levers. A rat is required to respond three times on the left lever in the presence of the left cue light. When this requirement is met, the left cue light goes off and the right cue light comes on. Three responses on the right lever produce a food pellet. The experimenter can terminate the program at any time by pressing any key on the computer keyboard. Termination causes the houselight to come on, causes the cue lights to go off, and

Table 1

BASIC Program for a Chained Fixed-Ratio Fixed Ratio Schedule Using a Commodore 64 Computer

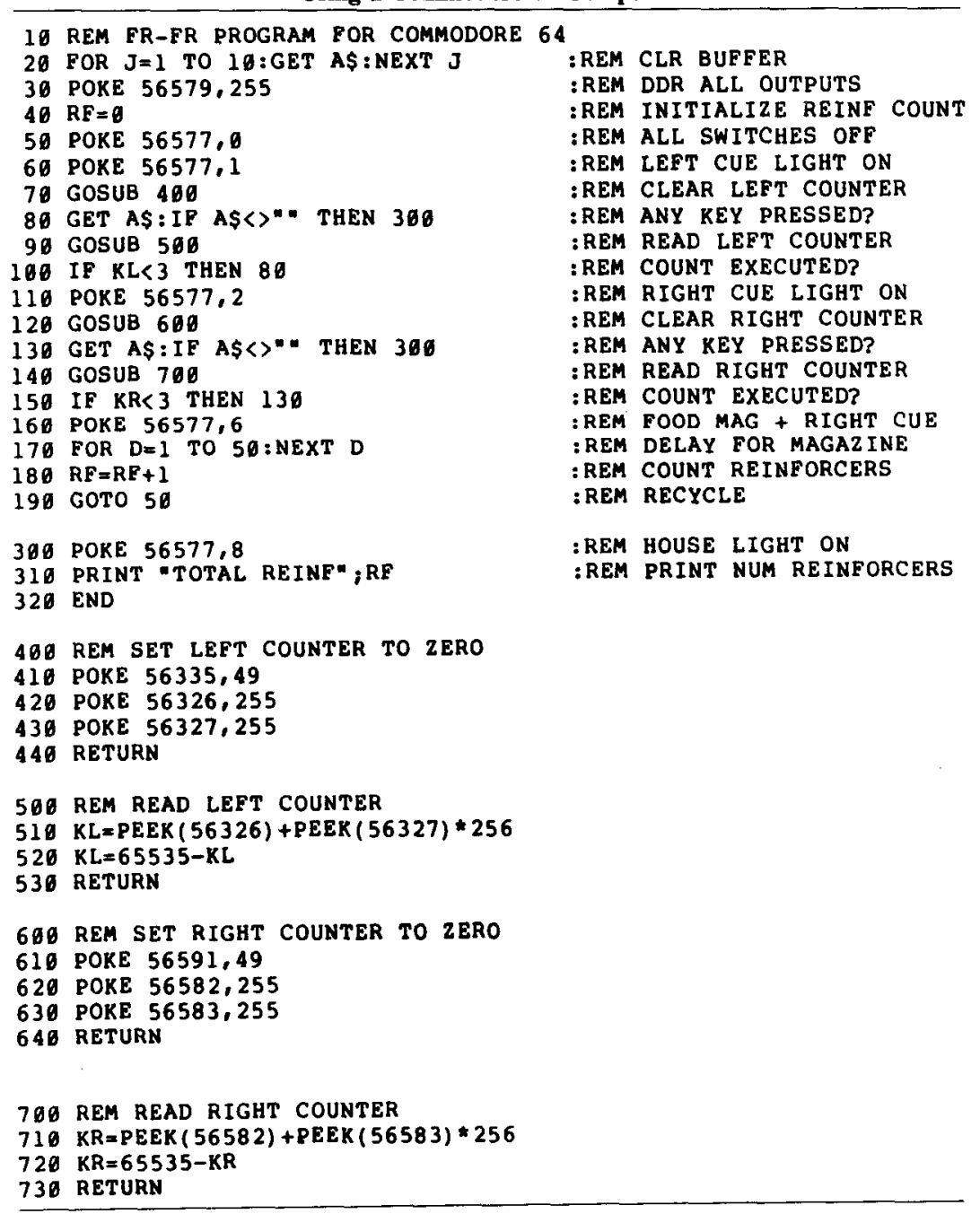


produces a program output which lists the total number of reinforcers received.

The line-by-line BASIC program for this experiment is shown in Table 1. With the studs numbered from 0 to 7 (from right to left on the I/O panel), the following locations are used in the program: 0 , left cue light; 1 , right cue light; 2, food magazine (pellet delivery); and 3, houselight.

The parameters of the schedule can easily be modified by changing the following: in Line 100 , insert new response requirement on left lever in place of " 3 ," and in Line 150, insert new response requirement on right lever in place of " 3 ."

A number of references supply comprehensive explanations of the computer, interfacing, and circuitry (Coffron, 1984; De Jong, 1980, 1984; Georgiou, 1984; Hildon, 1985; O'Dell, 1983). Also, the Commodore 64 Programmer's Reference Guide (Commodore Business Machines, 1984) provides an inexpensive source of valuable information.

\section{REFERENCES}

Coffron, J. W. (1984). The Commodore 64 connection. Berkeley, CA: Sybex.

COMmodore Business Machines (1984). Commodore 64 programmer's reference guide. Wayne, PA: Author.

De Jong, M. L. (1980). Programming and interfacing the 6502, with experiments. Indianapolis: Sams.

DE JoNG, M. L. (1984). Assembly language programming with the Commodore 64. Bowie, MD: Brady.

GEORGIOU, V. J. (1984). Commodore 64 interfacing blue book. Goleta, CA: Microsignal.

HILDON, K. J. (1985), The complete Commodore inner space anthology. Milton, OR: Transactor.

JACKSON, D. E., \& O'DeLl, J. W. (1985). The VIC-20 computer and an interface system for controlling operant chambers. Behavior Research Methods, Instruments, \& Computers, 17, 406-408.

O'DELL, J. W. (1983). TRS-80 as a controller: An introduction to interfacing. Peterborough, NH: Green Books.

(Revision accepted for publication March 3, 1986.) 